\title{
In situ neutron diffraction studies of operating MGA thermal storage materials
}

\author{
Heber Sugo ${ }^{1}$, Erich Kisi ${ }^{1,}$, James Bradley ${ }^{1}$, Thomas Fiedler ${ }^{1}$, and Vladimir Luzin ${ }^{2}$ \\ ${ }^{1}$ School of Engineering, The University of Newcastle, Callaghan, NSW 2308, Australia \\ ${ }^{2}$ Institute of Materials Engineering, Australian Nuclear Science and Technology Organisation, Locked Bag 2001, \\ Kirrawee DC, NSW 2232, Australia
}

Received: 15 January 2017 / Received in final form: 30 June 2017 / Accepted: 27 July 2017

\begin{abstract}
Miscibility Gap Alloys (MGA) store energy as the latent heat of fusion of a discrete component within an engineered multicomponent microstructure. The thermal energy density is augmented by the associated sensible heat of the whole alloy. MGA microstructures are organised so that the low melting point component, acting as a Phase Change Material (PCM), is present as dispersed, non-intersecting particles within a continuous 3 -dimensional solid matrix of a higher melting point component. The primary advantages of such an arrangement are the high thermal conductivity, 60-200 W/m K and high energy density $(0.25$ $1.26 \mathrm{MJ} / \mathrm{L}$ ). Long service life, low maintenance and the resulting simplified infrastructure offered by the ability of the MGA storage materials to exchange heat via conduction alone are further notable benefits. Unlike sensible heat storage materials, temperature measurement alone cannot indicate the state-of-charge of PCM storage since the temperature remains relatively constant throughout the melting or freezing transition. The work reported here addresses this issue through the use of in situ neutron diffraction to determine the relative volume fractions of solid (crystalline) and liquid PCM within the MGA material as a function of varying temperature conditions. The aim is to provide calibration data for state-of-charge thermal models of PCM storage systems. Preliminary analysis of the neutron diffraction results and associated thermal measurements from a series of experiments carried out using the KOWARI diffractometer at the Australian Nuclear Science and Technology Organisation are presented in this work.
\end{abstract}

\section{Introduction}

The intermittency of the Sun's solar resource represents a major challenge to the dispatchability of renewable energy generated from concentrated solar thermal power plants. Thermal storage materials and associated engineering approaches for storing and retrieving thermal energy have become important fields of research and development. There are numerous reviews on Thermal Energy Storage (TES) materials [1,2]. The two foremost methodologies for directly storing thermal energy as heat are "sensible" heat media or the use of Phase Change Materials (PCMs) to utilize the large amount of thermal energy absorbed/ released with changes of state such as melting/freezing. These enthalpy changes with variation in temperature are idealized in Figure 1.

In sensible energy storage, the specific heat capacity of a material is exploited to store and release thermal energy. This requires the TES material to undergo large

\footnotetext{
* e-mail: Erich.Kisi@newcastle.edu.au
}

temperature swings to store significant amounts of energy and during heat extraction the temperature of the working fluid decreases with time which has a negative impact on the output efficiency of the power cycle. The other approach, using PCM, is often also referred to as Latent Thermal Energy Storage (LTES). PCM yield higher energy densities than sensible heat media and offer the advantage that a large proportion of the energy is delivered or absorbed over a narrow temperature range as indicated in Figure 1. This near isothermal heat delivery has significant advantages for plant operational efficiency however it poses a major challenge as to how to evaluate the state-of-charge of the LTES. For sensible heat storage, the usual technique is to measure the temperature of the media. This is a poor indicator of stored energy in PCM systems as demonstrated in Figure 1. When the block temperature is in the latent heat range $\left(T_{\text {melt }}\right)$ the energy content of the block could be anywhere from approximately $12-88 \%$ of the maximum capacity.

A variety of PCM have been proposed including waxes, inorganic salts and metals $[3,4]$ with extensive reviews of PCM having been published [5-7]. However, with the 
Table 1. Properties of some MGA thermal storage materials [15]. ${ }^{\mathrm{b}}$

Type (active phase-matrix) Melting temperature of active phase $\left({ }^{\circ} \mathrm{C}\right)$ Thermal conductivity $(\mathrm{W} / \mathrm{m} \mathrm{K})$ Energy density $(\mathrm{MJ} / \mathrm{L})^{\mathrm{a}}$

\begin{tabular}{lrrl}
\hline Sn $50 \%-\mathrm{Al}$ & 230 & 120 & 0.43 \\
$\mathrm{Zn} 50 \%-\mathrm{C}$ & 420 & 70 & 0.65 \\
$\mathrm{Mg} 50 \%-\mathrm{Fe}$ & 650 & 100 & 0.58 \\
$\mathrm{Cu} 50 \%-\mathrm{Fe}$ & 1085 & 200 & 1.2 \\
$\mathrm{Si} 50 \%-\mathrm{SiC}$ & 1410 & 75 & 2.5 \\
\hline
\end{tabular}

a All values include $100^{\circ} \mathrm{C}$ of sensible heat capacity.

b A convenient graphical method of displaying the thermal properties of MGA was pioneered in earlier work and may be consulted for further comparison with other storage materials [14].

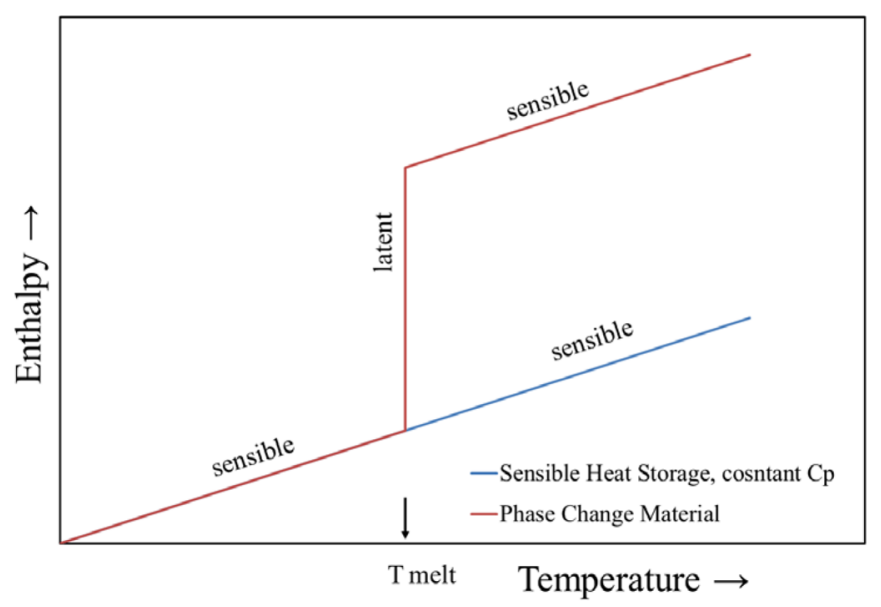

Fig. 1. Idealized enthalpy content with changes in temperatures for sensible and latent heat storage materials (adapted from [8]).

exception of Steinmaurer et al. [8], little attention has been paid to evaluating the state-of-charge for PCM systems. The general approach has been to do an input/output energy balance $[9,10]$ but in an operational plant with many charge/discharge cycles this is susceptible to cumulative errors. Steinmaurer et al. investigated the changes in volume/pressure and damping properties of wax type PCM to determine enthalpy content by estimating the solid: liquid volume ratios. Both forms were successful although void formation on solidification affected the volume/ pressure results. Steinmaurer concluded that the principle of wave propagation was the most promising, as it was applicable to a large range of PCM systems with low implementation costs.

This experimental study pioneers the use of in situ neutron diffraction to determine the spatial distribution and volumetric fraction of the solid/liquid phase change component in Miscibility Gap Alloys (MGA) under controlled temperature distributions. MGA is a new form of TES which incorporates small discrete metallic particles of a phase change component distributed in a rigid, high conductivity matrix[11]. The matrix is also metallic; however to avoid the formation of reaction by-products or preferential dissolution of a constituent both components are specially selected on the basis of limited or no miscibility with each other. This ensures constant enthalpy changes and longevity of the system. A further requirement of MGA is that the microstructure needs to be "inverted" to that formed in the "as-cast" condition. This is engineered by using specific particle size blends and volume fractions consolidated via powder metallurgy. Volumetric fractions of the phase change component are typically limited to $<50 \%$ to avoid percolation of the latent phase when molten. The advantages of MGA are the high thermal conductivity and energy density which combines the latent heat of the dispersed phase and the specific heat capacity of the whole alloy. Through selection of the alloy systems the heat delivery temperature can be specified. Several systems, composed of readily available metals, have been tested in the laboratory and are detailed in Table 1. MGA thermal storage materials are being developed at the University of Newcastle, Australia [12]. A cost comparison with established TES systems is presented elsewhere in this edition [13].

As part of the development of MGA storage materials thermophysical properties have been derived [14,15] enabling detailed thermal modelling of storage systems to be undertaken. From an operational point of view, data on the enthalpy content and spatial distribution of stored energy within the MGA storage systems is essential. Hence, MGA and other PCM based storage systems will require close monitoring of the energy budget as well as thorough thermal modelling.

For these models to be accurate they require calibration by independently measuring the level and distribution of charge in the material, preferably by determining the proportions of solid and liquid PCM and their distribution. One method of doing this is by neutron diffraction which is sensitive to only the crystalline portion of a material $[16,17]$. In this work, neutron diffraction experiments were conducted with the KOWARI diffractometer at the Australian Nuclear Science and Technology Organisation (ANSTO) facilities located at Lucas Heights, Sydney. MGA samples were heated under controlled temperature gradients and the integrated intensity diffraction data was then correlated to the measured temperatures under static and dynamic conditions.

\section{Methodology}

For these investigations a MGA system with a low melting point eutectic phase change component, namely $64 \mathrm{Sn}-$ $36 \mathrm{~Pb}$ (wt.\%, m.p. $183^{\circ} \mathrm{C}$ ) was selected. The samples contained a $40 \%$ volume fraction of PCM with an upper bound thermal conductivity of $\sim 140 \mathrm{~W} / \mathrm{m} \mathrm{K}$. The continuous matrix material was commercially pure aluminium. 

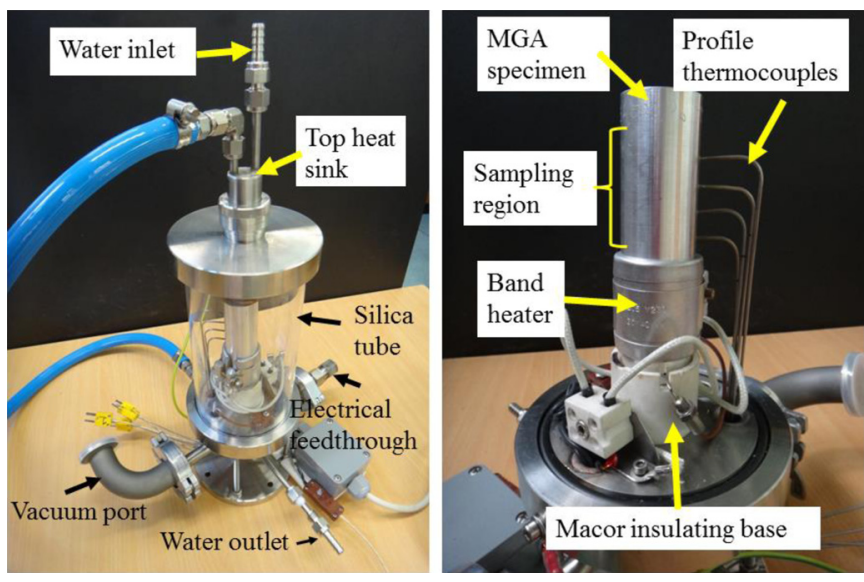

Fig. 2. MGA thermal gradient apparatus for neutron diffraction studies.

The MGA samples were prepared using a powder metallurgy technique followed by warm uniaxial pressing. The $64 \mathrm{Sn}-$ $36 \mathrm{~Pb}$ phase change component was supplied in shot form, approximately $2-3 \mathrm{~mm}$ in diameter with a slight tear drop shape. The powdered aluminium matrix material was a blend consisting of $<45 \mu \mathrm{m}$ and $230 \mu \mathrm{m}$ particles in a 60:40 mass ratio. Individual components were weighed and hand mixed. The batch was carefully added to an aluminium sleeve (6061 grade) which formed the outer shell of the sample. The nominal dimensions of the sleeve were $32 \mathrm{~mm}$ O.D. by $26 \mathrm{~mm}$ I.D. Solid aluminium top and bottom end caps were added to the sleeve to contain the powder during the pressing stage. The sleeves were then inserted in the warm press die and compacted at $100 \mathrm{MPa}$. The temperature was increased to $20^{\circ} \mathrm{C}$ below the melting point of the phase change component and held for $45 \mathrm{~min}$. Pressure was then released and the specimen heated to $300^{\circ} \mathrm{C}$ for $2 \mathrm{~h}$ to promote sintering of the matrix material. Finally the samples were machined to yield a $30 \mathrm{~mm}$ diameter $\times 110 \mathrm{~mm}$ high sample and four $1.5 \mathrm{~mm}$ diameter holes were drilled at $10 \mathrm{~mm}$ intervals to incorporate the thermocouples for temperature measurements.

A special apparatus was designed to impose a controlled temperature gradient on the MGA sample during the in situ neutron diffraction studies. This arrangement is shown in Figure 2 and consists of a $200 \mathrm{~W}$ band heater clamped to the lower portion of the specimen whilst a water cooled copper plate is used as the top heat sink. Macor spacers of different thicknesses were used as thermal resistors, between the sample and the top heat sink, to adjust the steepness of the temperature gradient within the diffraction region. To eliminate convection losses and maximize 1-dimensional heat flow, the specimen was enclosed within a $100 \mathrm{~mm}$ diameter fused silica tube fitted with stainless steel top and bottom flanges forming a vacuum chamber which was evacuated to $<10^{-4} \mathrm{~Pa}$ during operation. The fused silica material allowed visual alignment with minimal interference to the diffracted intensities due to its high neutron transparency. Water cooling was provided to the bottom flange below the specimen support. A programmable proportioning integrating differentiating temperature controller was used to regulate the band heater temperature. The four Type $\mathrm{K}$ thermocouples were monitored with a Datataker DT80 logger.
The diffractometer arrangement and thermal gradient apparatus is shown in Figure 3(a). A tightly collimated neutron beam was positioned in the centre of the MGA cylindrical sample yielding a gauge volume $10 \mathrm{~mm}$ deep $\times 10 \mathrm{~mm}$ wide $\times 1 \mathrm{~mm}$ high. Beam parameters consisted of thermal neutrons $(\lambda=1.73 \AA)$ with nominal intensity of $10^{7}$ neutrons $\mathrm{cm}^{-2} \mathrm{~s}^{-1}$. The detector was placed at a diffraction angle of $2 \theta \approx 90^{\circ}$ position to enable the reflections from the $\mathrm{Al}$ (311), Sn (312) and $\mathrm{Pb}$ (400) planes to be detected if present. A typical detector output showing the $\mathrm{Al}$ and $\mathrm{Sn}$ reflections is given in Figure 3(b).

Samples were scanned with the neutron beam in the vertical direction using fine steps to establish the distribution of the solid constituents. Once the room temperature distribution maps were established, the sample temperature was increased until the $\mathrm{Sn}$ and $\mathrm{Pb}$ intensity disappeared. The temperature was then held static and the sample scanned again in the vertical direction to obtain the spatial distribution of the solid/ liquid PCM. Dynamic tests were also performed by fixing the scan position on the sample and driving the melt/ solidification front through this region using heating/ cooling ramps to simulate charge-discharge cycles.

Neutron diffraction data was analysed by converting detector counts to intensity- $2 \theta$ plots and fitted with Gaussian profiles to extract integrated diffracted intensity. Only the diffracted intensities are presented in this preliminary report. Corrections for structure, Lorentz and multiplicity factors according to standard methods for single peak quantitative phase analysis are required to yield actual mass and volume fractions of each component [17]. These will be presented in a more detailed report.

\section{Results and discussions}

A typical room temperature scan, for the $40 \%$ by volume $(\mathrm{Sn}+\mathrm{Pb})$ in aluminium, is indicated in Figure 4(a). This shows the distribution of the three crystalline constituents; $\mathrm{Al}$ matrix, $\mathrm{Sn}$ and $\mathrm{Pb}$. The latter two constituents form the eutectic fusible phase. It may be observed that the distribution is not uniform along the length of the sample (relative position) due to the random packing of the $\mathrm{Sn}+\mathrm{Pb}$ eutectic particles within the matrix during the manufacture. Overall, a strong intensity is observed for the $\mathrm{Al}$ (311) reflection, followed by the Sn (312) and a weaker signal from the $\mathrm{Pb}(400)$ reflection. The poor observed intensities of the $\mathrm{Pb}$ (400) peaks were attributed to the low multiplicity factor provided by this family of planes; however, melting of the PCM can be established by the behaviour on the Sn alone. This is due to the eutectic reaction which on solidification simultaneously forms a mixture of $\mathrm{Sn}$ and $\mathrm{Pb}$ crystals.

\subsection{Static temperature gradients}

Once the room temperature distribution of the three crystalline components was established, a temperature gradient was induced in the sample to locate the melt front in the central portion of the sample. Scans were then recorded along the entire neutron diffraction gauge region. These 

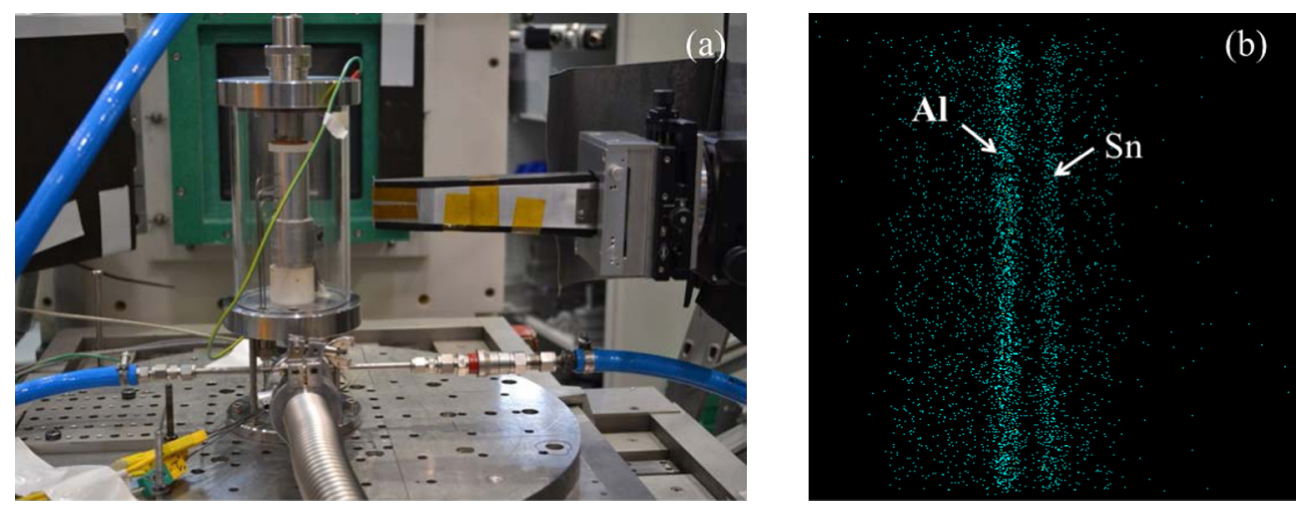

Fig. 3. (a) Specimen chamber mounted on KOWARI diffractometer and (b) diffraction pattern as observed on detector.
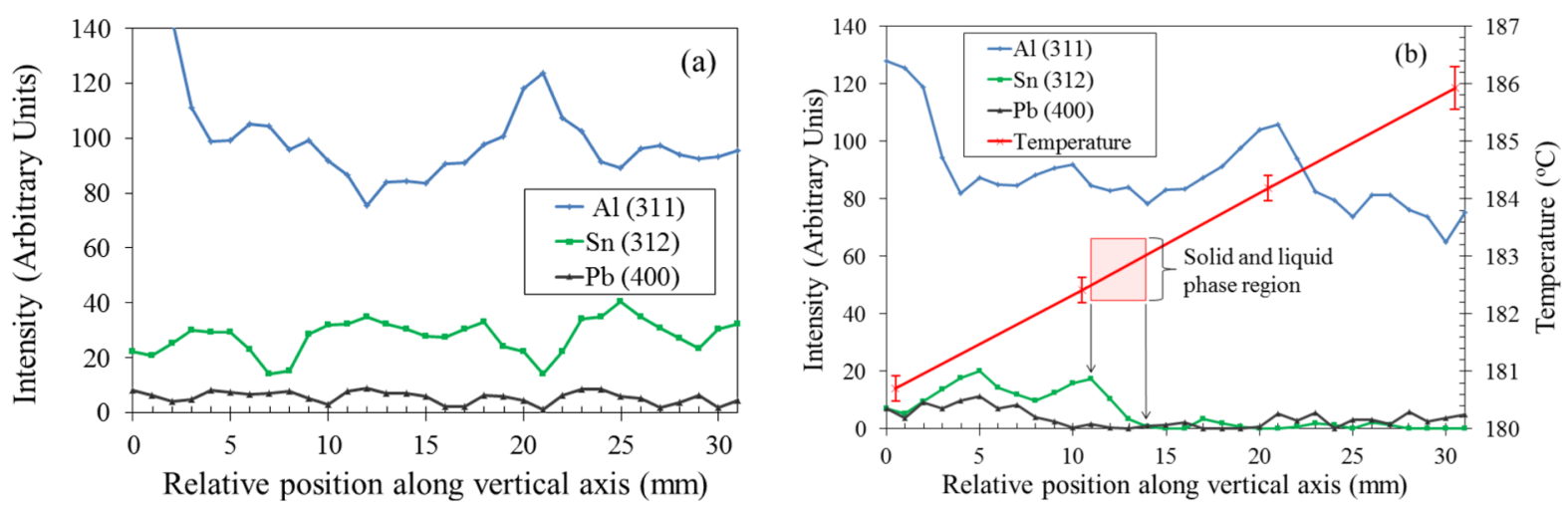

Fig. 4. (a) Intensity distribution of $\mathrm{Al}, \mathrm{Sn}$ and $\mathrm{Pb}$ at room temperature and (b) intensity plot under static temperature gradient.

observed intensities are presented in Figure 4(b) together with the measured temperatures along the sample length. Each temperature data point represents the mean and one standard deviation of 4000 readings taken during the scans. In this preliminary analysis, due to steady-state conditions, a simple linear interpolation has been used to represent the temperature gradient along the sample length.

Comparisons of Figure 4(a) and (b) show a similar trend for the distribution of $\mathrm{Al}$ as a function of the relative vertical position with the minor variations being mainly associated with thermal expansion effects. In Figure 4(b), the Sn reflections start to reduce in intensity after the $11 \mathrm{~mm}$ relative position and approach zero after the $14 \mathrm{~mm}$ point. This is in contrast to the strong Sn reflections observed at room temperature scan shown in Figure 4(a). This $\sim 3 \mathrm{~mm}$ long region corresponds to the coexistence $\mathrm{Sn}$ in both crystalline and liquid states. The observed temperature range for this transition, $\sim 182.4-183.4^{\circ} \mathrm{C}$, is in good agreement with the known eutectic melting point of $183^{\circ} \mathrm{C}$. The trend for the $\mathrm{Pb}$ component is not as clear due to the factors noted above however, a reduction in intensity can still be observed in the $11-14 \mathrm{~mm}$ positions. At positions $>14 \mathrm{~mm}$, the low intensities indicate that $\mathrm{Sn}$ and $\mathrm{Pb}$ are completely molten and the MGA is in the fully charged condition.

\subsection{Dynamic temperature gradients}

The relative intensity- $2 \theta$ plot for the crystalline phases in the MGA sample undergoing cyclic testing is shown in Figure 5 together with the temperature profile experienced in the beam volume plotted on the secondary ordinate axis. The intensity of the $\mathrm{Al}$ (311) reflection has again only minimal temperature effects. The melting/solidification behaviour is clearly shown in the step-like nature of the $\mathrm{Sn}$ (312) relative intensity. Regions where both solid and liquid Sn coexist are indicated by the boxed regions superimposed on the temperature plot. The first solidification transition, at a cooling rate of $1{ }^{\circ} \mathrm{C} / \mathrm{min}$, starts at $180^{\circ} \mathrm{C}$ and is complete by $174^{\circ} \mathrm{C}$. The second solidification cycle, at $0.5^{\circ} \mathrm{C} / \mathrm{min}$, starts at $181^{\circ} \mathrm{C}$ and ends at $179^{\circ} \mathrm{C}$. This indicates that the slower cooling rate reduces slightly the degree of undercooling required for nucleation and subsequent crystal growth. During the heating cycle, the intensity of the Sn diffraction peaks start to decrease once the melting point $\left(183^{\circ} \mathrm{C}\right)$ is reached and melts completely within a $4^{\circ} \mathrm{C}$ window. As noted above, the trend from the $\mathrm{Pb}$ intensities is not as clear.

The minor differences in the Sn mean intensities, between the first and second solidification cycles were also observed in other diffraction patterns. Generally, a higher cooling rate resulted in higher intensities. This is believed to arise due to the higher cooling rates yielding smaller, more numerous crystals in the beam volume. This technique, or the use of smaller PCM particles during the manufacture of the MGA material, is likely to improve the observed intensities as more crystals would be present in the beam volume.

The results from these preliminary in situ neutron diffraction studies indicate that for steady state conditions the sharpness of the melt/solid interfaces for this MGA 


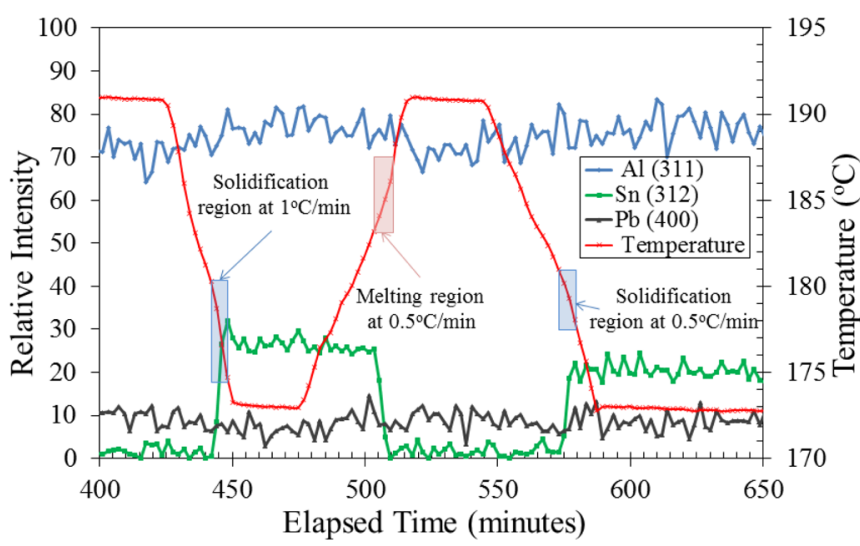

Fig. 5. Relative intensity (left hand scale) for MGA sample during temperature cycling. The local temperature experienced by the material in the beam volume is shown in red, refer to right hand scale.

system is in the order of $1^{\circ} \mathrm{C}$ over a distance of $3 \mathrm{~mm}$. In the dynamic tests, no time lag could be observed during the charging stage once the melting point temperature was reached and minimal undercooling was required for the release of latent heat on solidification. This confirms that temperature alone is not a good indicator of the state-ofcharge in PCM systems and highlights the need for carefully calibrated thermal models.

\section{Conclusions}

In situ neutron diffraction was found to be a successful technique to investigate the spatial distribution of solid and liquid PCM in MGA samples. The sharp melt/ solidification transition in this highly thermal conductive material was able to be identified and measured. This quantitative data can be used to directly estimate the state-of-charge of MGA thermal storage materials in order to develop calibrated thermal models for larger, operational systems. For the specific system investigated, refinements in the technique include the use of smaller PCM particles to yield higher diffracted intensities by increasing the number of crystals in the neutron gauge volume. The use of in situ neutron diffraction techniques to quantify the solid/liquid distribution of the latent phase in MGA can also be extended to investigate other PCM.
This work is part of an on-going investigation funded through an ARC Discovery Project (DP150101257). In addition, the authors would like to thank the Bragg Institute at ANSTO for providing beam time on the KOWARI diffractometer Proposal P4999. Finally, the authors would also like to extend their thanks to the staff in the School of Engineering at the University of Newcastle. A special thanks to Mr. Dean Ferry for the fabrication of the thermal gradient apparatus and assistance with sample preparation.

\section{References}

1. S. Kuravi, J. Trahan, D.Y. Goswami, M. Rahman, E.K. Stefanakos, Progr. Energy Combust. Sci. 39, 285 (2013)

2. T. Kousksou, P Bruel, A Jamil, T. El Rhafiki, Y. Zeraouli, Sol. Energy Mater. Sol. Cells 120, 59 (2014)

3. T. Bauer, D. Laing, R. Tamme, Adv. Sci. Technol. 74, 272 (2010)

4. Z. Kha, Z Khan, A. Ghafoor, Energy Convers. Manag. 115, $132(2016)$

5. B. Zalba, J.M. Marin, L.F. Cabeza, H. Mehling, Appl. Therm. Eng. 23, 251 (2003)

6. M.M. Farid, A.M. Khudhair, A.K.S. Razack, S. Al-Hallaj, Energy Convers. Manag. 45, 1597 (2004)

7. T. Nomura, N. Okinaka, T. Akiyama, ISIJ Int. 50, 1229 (2010)

8. G. Steinmaurer, M. Krupa, P. Kefer, Energy Procedia 48, $440(2014)$

9. R. Bayón, E. Rojas, L. Valenzuela, E. Zarza, J. León, Appl. Therm. Eng. 30, 2634 (2010)

10. X. Xiao, P. Zhang, Heat Transf. Eng. 35, 1084 (2014)

11. H.O. Sugo, E.H. Kisi, D.T. Cuskelly, Appl. Therm. Eng. 51, 1345 (2013)

12. Australian Patent No. AU2013334479, Alloys with inverse microstructures

13. A. Post, A. Rawson, H. Sugo, D. Cuskelly, J. Bradley, M. Copus, E. Kisi, Renew. Energy Environ. Sustain. (2017) (this edition)

14. A.J. Rawson, H.O. Sugo, E.H. Kisi, in Proceedings 52nd Conference of the Australian Solar Council, Melbourne, Australia (2014)

15. A.J. Rawson, PhD thesis, University of Newcastle, Newcastle, Australia, 2016

16. G.E. Bacon, Neutron Diffraction, 3rd edn. (Clarendon Press, Oxford, 1975)

17. E.H. Kisi, C.J. Howard, Applications of Neutron Powder Diffraction (Oxford University Press, Oxford, 2008)

Cite this article as: Heber Sugo, Erich Kisi, James Bradley, Thomas Fiedler, Vladimir Luzin, In situ neutron diffraction studies of operating MGA thermal storage materials, Renew. Energy Environ. Sustain. 2, 34 (2017) 